\title{
A Simple and Reliable Method for the Evaluation of the Exposed Field Near the GSM Antenna
}

\author{
Algenti Lala ${ }^{1}$,Bexhet Kamo ${ }^{1}$, Vladi Kolici ${ }^{1}$, Shkelzen Cakaj ${ }^{1}$, \\ ${ }^{1}$ Faculty of Information Technology \\ Polytechnic University of Tirana \\ Tirana, ALBANIA \\ ${ }^{2}$ Faculty of Electrical and Computing Engineering \\ Prishtina University \\ Prishtina, KOSOVO
}

\begin{abstract}
The objective of this paper is to present a simple, accurate and very efficient method for the evaluation of the field in the vicinity of GSM antennas of the radio base-station in urban areas. The method is based on the replacement of the antenna panel with a group of discrete source emitters. A geometrical approximation is used for the evaluation of the environment's influence also. The calculated results are compared with results taken from the use of NARDA SRM 3000 measuring equipment. The presented method could be successfully used for the exposure evaluation of the electromagnetic field emitted by GSM antennas of the basestation in urban areas.
\end{abstract}

Keywords-evaluation; electromagnetic field;near field; NARDA SRM 3000; base-station

\section{INTRODUCTION}

The fast deployment of radio communicating systems, especially in the mobile technology, has raised concerns in regards to the effects of electromagnetic field emitted by these systems on human health. This paper aims to evaluate the electromagnetic field emitted by the antennas of the basestations.

The evaluation of the field in the vicinity of the BS can be done by using simple calculating methods and simulations; both approaches are complementary and allow for the exploration of the whole space around the antenna. The simulation method is of great interest as it can be used for verifying the results of practical measurements and for extrapolating these results beyond the allowed space, where measurements cannot be done.

This paper presents a brief examination of the methodologies used for predicting, in the context of verifying reference levels of power density near antenna panels of BS which are usually used in mobile telephony in GSM bands $900 \mathrm{MHz}$ and $1800 \mathrm{MHz}$.

The exposure to BS antennas in free space has been studied in references [1], [2], [3], [4] and in reference [5] for urban environment until now. We aim to solve the problem of evaluating the exposure to the near field in the urban environment. In the zone of far field, which is defined as the "zone of antenna field where the spread is in substance independent from the distance from the antenna" [6], the EM field can be calculated relatively easy since all the required information is in the amplifying model of the antenna and the emitted power.

If the antenna has a maximum dimension $\mathrm{D}$, the field in the distant zone, usually is taken as the radial distance from the source $R=2 D^{2} / \lambda$, where $\lambda$ is the wavelength. For distances smaller than R, in the zone of the near field, the EM field has a complex form, which is very difficult to evaluate. For a GSM antenna panel of the BS $(\mathrm{D}=2 \mathrm{~m})$ the border between the near field and the far field is in the distances of $\sim 10 \mathrm{~m}$ and $\sim 20 \mathrm{~m}$ for GSM $900 \mathrm{MHz}$ and GSM $1800 \mathrm{MHz}$ respectively. The conditions of exposure are completely different in the urban environment from the ones in the free space. The "rigorous" numeric modeling techniques, such as the method of moments (MoM) or the finite-difference time-domain method (FDTD), are used extensively in the study of the field in the proximity of the antenna. The most common issue related to the application of these methods is to know the geometry of the $\mathrm{BS}$ antenna. In addition to the issue mentioned above, when the exposure in the urban environment is modeled, the dimensions of the area of study might be very large compared with the wavelength; as a result the time needed for the analysis is unacceptably long. We definitively need simpler and more reliable calculating methods for forecasting the exposure of EM field in the urban environment.

This paper is organized as follows. The first section goes over the need for a simple, and practical method to evaluate the field in the nearby of the radio base stations. Section II describes the modeling and geometry of the antenna. Section III is referred to proposed theoretical model for the evaluation of the far field. Section IV presents results and discussions. Section V draws the conclusions.

\section{THE MODELING AND GEOMETRY OF THE ANTENNA}

The numeric calculations have been performed for a typical BS antenna. We took into considerations the Katherine 80010670,80010671 and 80010672 [7], type for a range of frequencies between $870 \mathrm{MHz}$ and $960 \mathrm{MHz}$, and $1710 \mathrm{MHz}$ and $1880 \mathrm{Mhz}$. The emitting model of this antenna is characterized by a $(\mathrm{G}) 17.5 \mathrm{~dB}$ amplification, an opening (aperture) of $3 \mathrm{~dB} 7^{\circ}$ and $65^{\circ}$ in the vertical plan (plan E) horizontal plan (plan $\mathrm{H}$ ), respectively. The model is in the shape of group of dipoles positioned in front of a reinforcing 
reflector with dimensions: $2 \mathrm{~m}$ long and $40 \mathrm{~cm}$ wide, with angles for limiting the main ray. According to the reflecting theory[8], the dividing distance between the reflector and the group of dipoles is set at $\lambda / 4$. The number of dipoles, which depends from the desired amplification, and dividing distance between them is calculated according to [8].

$$
\Theta_{h}=2\left|\theta_{m}-\theta_{h}\right|=2\left|90^{\circ}-\cos ^{-1}\left[\frac{\lambda}{2 \pi d}\left(-\beta \pm \frac{2.782}{N}\right)\right]\right|
$$

Where $\Theta_{h}$ is half the power of the ray width, $\theta_{m}$ is the maximum value of the group factor which is set at $90^{\circ}, \theta_{h}$ is the point with half power, $\lambda$ is the wavelength (for $\mathrm{f}=947.5$ $\mathrm{MHz}$ which is the the middle frequency of the downlink band in GSM), $N$ is the number of elements, $\beta$ is the shift of the phase between the elements and $d$ is the distance between them.

In the vertical plan (plan $E$ ), the distance between the poles (center-center) which are along the $\mathrm{Z}$ axis and with the same distance, can be calculated by (1). Considering the effect of the reflector, by putting the dividing distance between elements at $d_{z} \leq 3 \lambda / 4$, which is given at $22 \mathrm{~cm}$, the number of $N_{z}$ elements can be calculated in order to achieve a half power of the ray width $7^{\circ}$ by considering $\beta=0$ and the point of half power at $\theta_{h}=3.5^{\circ}$; this corresponds with eight elements.

In the horizontal plan (plan $H$ ), the dividing distance between $d_{y}$ dipoles which are encountered along the $\mathrm{Y}$ axis can be calculated in the same way from (1) by putting $d_{y} \leq \lambda / 2$ which is given at $11 \mathrm{~cm}$. The number of $N_{y}$ elements can be calculated in order to gain a ray width of $65^{\circ}$ with half power. Considering the point of half power at $\theta_{h}=32.5^{\circ}$, which coresponds with two elements. As a result the group of dipoles is formed by $8 \times 2$ dipoles half wavelength $(\lambda / 2)$ along the $Z$ axis and $\mathrm{Y}$ axis, as shown in Fig.1.

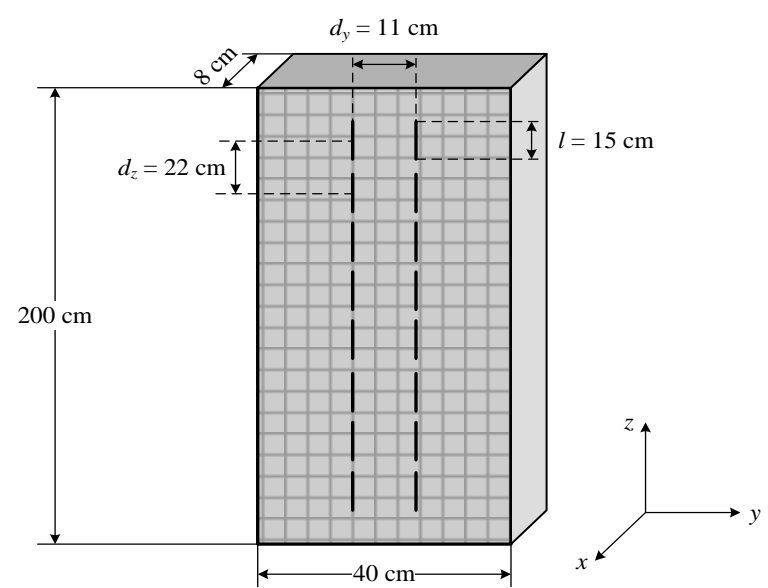

Fig. 1. The front and side view of the projected antenna

By using the sub-antenna description, the antenna model is valid for the near and distant fields for the whole antenna. Because of the distribution and reflections, the environment has some influence in the power of the field. In order to take into account the possible distribution, the evaluation of the field near the antenna is done by using the reflections theory. The 'ray tracing method' can be used in the distant field of these sub-antennas

\section{THE METHOD FOR EVALUATING THE NEAR FIELD OF AN ANTENNA}

Modeling of the electromagnetic field near the radio base stations is a way to evaluate and define the excluded zones near these stations. The selection of an appropriate model is important in order to have a good estimation of the levels of the radiation.

In the references [8], [9], [10], there are many models for the definition of the zones of the near field as well as the far field. Attention must be paid to the fact that in the zones of the near field, the levels of the radiation depends not only on the distance from the antenna but also on the movement along the vertical axis, whereas in the case of the far field the levels depend only on the distance not on the movement along the vertical axes. The models of the far field aim towards simple formulations and based on them numerical methods can be applied which make possible the estimation of the electromagnetic field in a short time and with modest computer processing power.

The proposed model is based on the model "Far-field Gain-based" [11] and eq. (2). This model provides a simple and efficient method for the evaluation of the levels of the electromagnetic field radiated by the antennas of the radio base stations with uniform groups of cells in the zone of the near field and the ones of the far field. The above is achieved in two steps:

In the first step, electrical intensity of the antenna is calculated by combining the radiation of the far field of the antenna elements, and the group factors, by accepting that the antennas of the radio base stations are a uniform group of cells. Modeling the antenna with N source cells is shown in Fig. 2.

$$
E(d, \theta, \varphi) \approx\left|\sum_{i=1}^{N} \frac{\sqrt{30 P_{i n} G_{e}\left(\theta_{i}, \varphi_{i)}\right)}}{d_{i}} e^{-j \phi_{i}} u\left(\theta_{i}, \varphi_{i}\right)\right|, d>3 \lambda(2)
$$

Second step as eq. (2):

$$
\begin{array}{r}
G_{e}\left(\theta_{i}, \varphi_{i}\right) \approx \frac{G_{M} D_{V e}(\theta) D_{H e}(\varphi)}{N} \\
\phi_{i}=(i-1) \phi+\frac{2 \pi d_{i}}{\lambda}
\end{array}
$$

Where $\mathrm{N}$ is the number of radiating cells, $\left(\mathrm{d}_{\mathrm{i}}, \theta_{\mathrm{i}}, \varphi_{\mathrm{i}}\right)$ are spherical coordinates of the $\mathrm{i}$-th element up to the $\mathrm{N}$-th one, $\mathrm{P}_{\text {in }}$ is total radiated power by a given group, $\mathrm{G}_{\mathrm{e}}\left(\theta_{\mathrm{i}}, \varphi_{\mathrm{i}}\right)$ is amplification of the radiating element, $d_{i}$ is distance from the $i$ th element, $u(\theta i, \varphi i)$ is unit vector of the $\mathrm{i}$-th element, $\lambda$ is wave length , GM maximal gain of the antenna, $D_{\mathrm{Ve}}(\theta), \mathrm{D}_{\mathrm{He}}(\varphi)$ are the models of the radiating element in the vertical and horizontal plan and $\Phi_{\mathrm{i}}$ is the differences of the phases between the coefficients of the radiating element. 


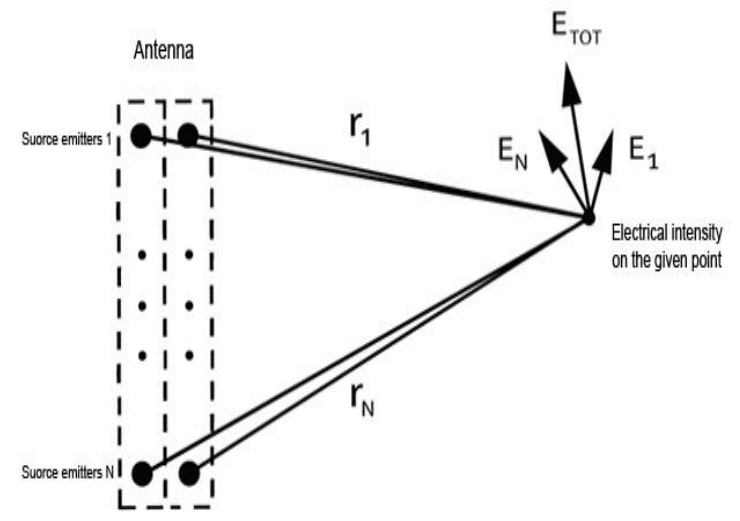

Fig. 2. Modeling the antenna with $\mathrm{N}$ source cells

In this paper, reference is made to Kathrein antennas specifically to the models, 80010670, 80010671 and 80010672. The Kathrein model 80010671 [7] is used for the spectrums 900/1800/2100 MHZ. The intensity of the electric field calculated for each of the elements (cells) of the antenna is vectorial and it can be projected according to the axis $\mathrm{x}, \mathrm{y}$, $\mathrm{z}$, and obtain the respective components for each of the axes. The electric and magnetic fields in the Cartesian coordinates are composed of each of the three components Ex, Ey and Ez for each and every frequency the same is valid for the magnetic field with respective $\mathrm{Hx}, \mathrm{Hy}$ and $\mathrm{Hz}$ components [8] as (5):

$$
E_{\text {Resultant }}=\sqrt{\left(E_{x}\right)^{2}+\left(E_{y}\right)^{2}+\left(E_{z}\right)^{2}}
$$

We propose for the modeling of the base antennas the following:

1- The use of the "Far-field Gain-based" model in which the intensity of the electric field is calculated by the equation (2) with the approximation that $e^{-j \phi_{i}} u\left(\theta_{i}, \varphi_{i}\right)=1$. This approximation influents the accuracy of the model "Far-field Gain-based" for the near fields up to $15 \lambda$. This falls within the safety distances as defined by the standards.

The acquired equation is (6):

$$
E(d, \theta, \varphi) \approx\left|\sum_{i=1}^{N} \frac{\sqrt{30 P_{i n} G_{e}\left(\theta_{i}, \varphi_{i)}\right)}}{d_{i}}\right|
$$

2- The statistical study considered is "The worst case scenario the vectors $E_{x 1} E_{x 2} \ldots E_{x n}$ in the same phase as the $E_{y}$ and $E_{\mathrm{z}}$ ". This definition will lead to an overestimation of the electromagnetic field on the given point. The intensity of the electrical field in a given point (weight per frequency) near the antenna of a radio base station when the antenna is ThreeBand (900/1800/2100) and by considering the "vectors Ex1, Ex2 ...Exn in the same phase as the Ey and Ez" is as per the (7).
$\mathrm{E}_{\mathrm{Rfrequence}}$ - the electromagnetic field radiated by the antenna on the calculated point [13].

$$
E_{\text {Rfrequence }}=\sqrt{\left(\sum_{1}^{N}\left(E_{x}\right)^{2}+\sum_{1}^{N}\left(E_{y}\right)^{2}+\sum_{1}^{N}\left(E_{z}\right)^{2}\right.}
$$

Given that the behavior of the near field is much more complex than the distant field, it would be easier to include the space of the near field as forbidden zone for people. In practice, the space of the zone of near field would include an area with a radius of $20-40 \mathrm{~m}$ for a typical base-station antenna $2 \mathrm{~m}$ long (for this work we have limited the distance up to $4 \mathrm{~m}$ from the antenna panel center) is shown in Fig. 3 .

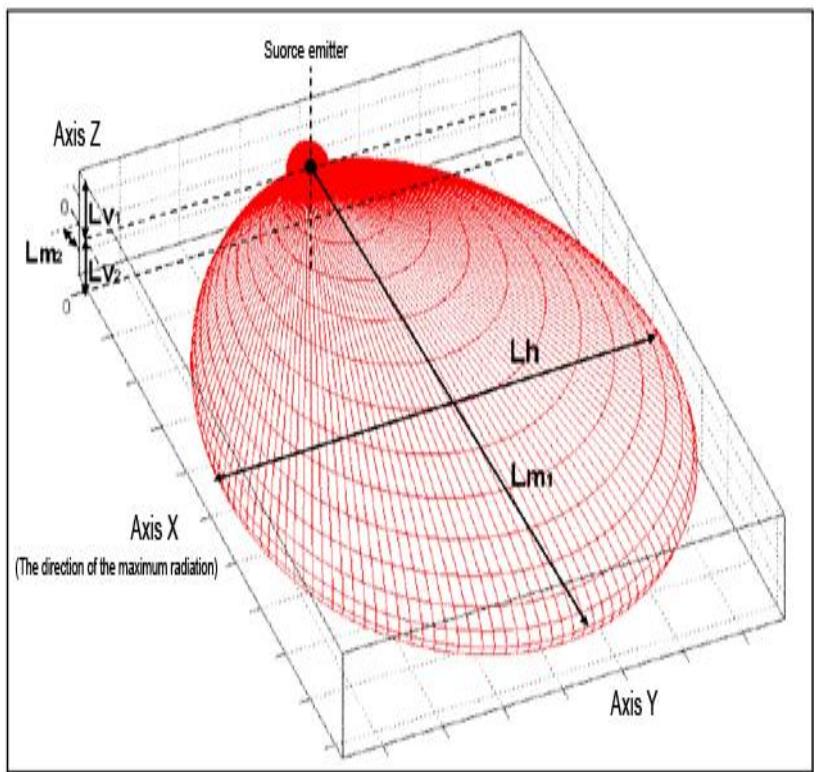

Fig. 3. The shape and dimension of a volume for a sector of the base station transmitter

\section{ANALYSIS OF THE RESULTS}

This section shows the results of our work. In order to check the accuracy of the calculating method presented, the approximate results are compared with the results from the measurement done with the NARDA SRM 3000 [12] measuring equipment. The value for a sample is achieved by an average of measurements that last 360 seconds. The sampling step in the $\mathrm{Z}$ axis is every $10 \mathrm{~cm}$ in the interval $\pm 1 \mathrm{~m}$ the middle of the antenna; the sampling step in the $\mathrm{X}$ axis is every $20 \mathrm{~cm}$ in the interval 0 to $4 \mathrm{~m}$. The sampling step in the $\mathrm{Y}$ axis is every $10 \mathrm{~cm}$ in the interval $\pm 1 \mathrm{~m}$ front and back. The results taken are shown in Fig.4 and Fig.5.

The results of the electric field in the vicinity of the antenna Katherine 80010671, are presented in Fig. 4. As it can be easily noted, the approximated results are in accordance with the results received from the analysis using a full wavelength. The continuous lines represent the simulated values. The dashed lines represent the measured values. 


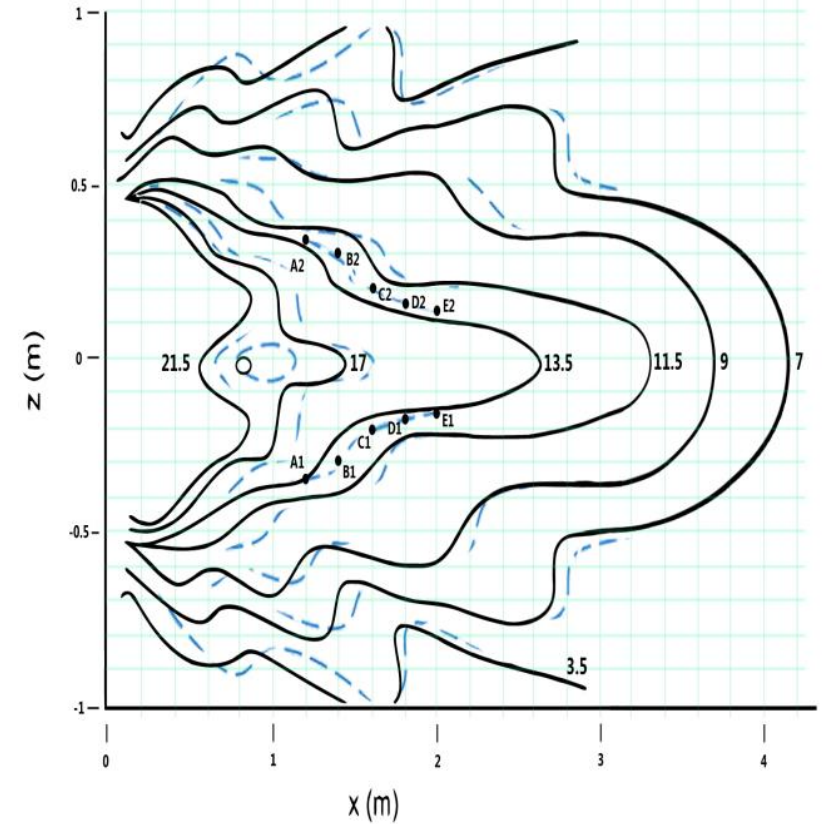

Fig. 4. The electric field in the vicinity as per $\mathrm{Z}$ direction

To simplify the comparison of the results between the computed values and the measured ones, we have prepared a table. Coordinates of the points A1, A2, B1, B2, C1, C2, D1, $\mathrm{D} 2$, E1, and $\mathrm{E} 2$ as per the $\mathrm{X}$, and $\mathrm{Z}$ axis are presented in Table I. In the last two columns are the results computed by applying the proposed method simulated in Matlab, and the measured values.

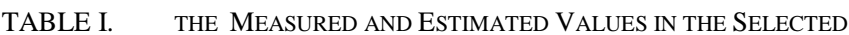
POINTS OF INTEREST AS IN FIG. 4

\begin{tabular}{lllll}
\hline Points & $\mathbf{X}(\mathbf{m})$ & $\mathbf{Z}(\mathbf{m})$ & $\mathbf{E}_{\text {estimated }}(\mathbf{V} / \mathbf{m})$ & $\mathbf{E}_{\text {measured }}(\mathbf{V} / \mathbf{m})$ \\
\hline $\mathrm{A}_{1}$ & 1.2 & -0.35 & 13.5 & 13.596 \\
$\mathrm{~A}_{2}$ & 1.2 & 0.35 & 13.5 & 13.227 \\
$\mathrm{~B}_{1}$ & 1.4 & -0.3 & 13.5 & 12.883 \\
$\mathrm{~B}_{2}$ & 1.4 & 0.3 & 13.5 & 12.842 \\
$\mathrm{C}_{1}$ & 1.6 & -0.2 & 13.5 & 12.810 \\
$\mathrm{C}_{2}$ & 1.6 & 0.2 & 13.5 & 12.810 \\
$\mathrm{D}_{1}$ & 1.8 & -0.15 & 13.5 & 13.245 \\
$\mathrm{D}_{2}$ & 1.8 & 0.15 & 13.5 & 13.245 \\
$\mathrm{E}_{1}$ & 2 & -0.10 & 13.5 & 13.348 \\
$\mathrm{E}_{2}$ & 2 & 0.10 & 13.5 & 13.270 \\
\hline
\end{tabular}

In Fig. 5 are selected the points A0, A1, A2, A3, B0, B1, $\mathrm{B} 2, \mathrm{~B} 3, \mathrm{C} 0, \mathrm{C} 1, \mathrm{D} 0, \mathrm{D} 1, \mathrm{E} 0$ and $\mathrm{E} 1$. The respective coordinates as per $\mathrm{X}$ and $\mathrm{Y}$ directions are presented in the Table II. In the last two columns of the table are introduced the results of the electromagnetic field, computed by using the proposed method simulated in Matlab, and the measured values of the electromagnetic field.

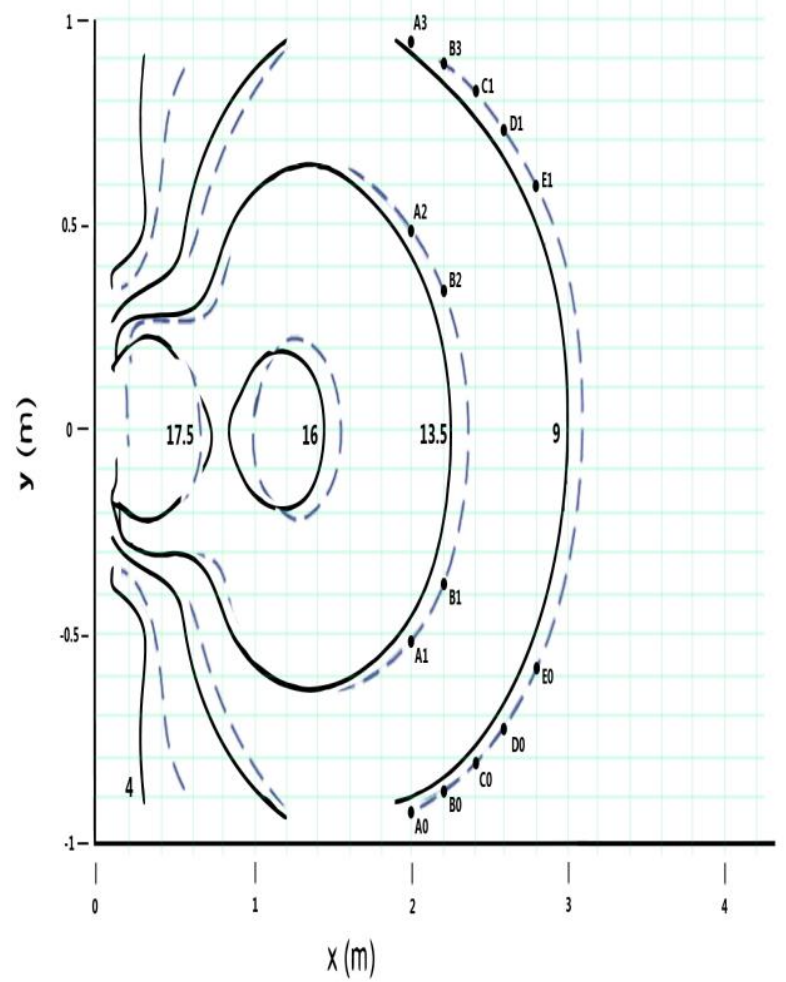

Fig. 5. The electric field in vicinity as per Y direction

TABLE II. THE Measured and Estimated VAlues IN THE SELECTED POINTS OF INTEREST AS IN FIG. 5

\begin{tabular}{lllll}
\hline Points & $\mathbf{X}(\mathbf{m})$ & $\mathbf{Y}(\mathbf{m})$ & $\mathbf{E}_{\text {estimated }}(\mathbf{V} / \mathbf{m})$ & $\mathbf{E}_{\text {measured }}(\mathbf{V} / \mathbf{m})$ \\
\hline A0 & 2 & -0.95 & 9 & 8.372 \\
A1 & 2 & -0.50 & 13.5 & 13.134 \\
A2 & 2 & 0.50 & 13.5 & 13.134 \\
A3 & 2 & 0.95 & 9 & 8.372 \\
B0 & 2.2 & -0.90 & 9 & 8.372 \\
B1 & 2.2 & -0.35 & 13.5 & 13.134 \\
B2 & 2.2 & 0.35 & 13.5 & 13.134 \\
B3 & 2.2 & 0.90 & 9 & 8.372 \\
C0 & 2.4 & -0.80 & 9 & 8.372 \\
C1 & 2.4 & 0.80 & 9 & 8.370 \\
D0 & 2.6 & -0.70 & 9 & 8.370 \\
D1 & 2.6 & 0.70 & 9 & 8.370 \\
E0 & 2.8 & -0.60 & 9 & 8.370 \\
E1 & 2.8 & 0.60 & 9 & 8.370 \\
\hline
\end{tabular}

As it can be noted by the above tables (TableI, and Table II) we can see a considerable approximation of the calculated values using the proposed method, and the measured ones in the same points. The error between the two is less than $10 \%$. 


\section{CONCLUSION AND FUTURE WORK}

This paper introduced a simple but very effective method in terms of calculations, for the evaluation of the electric field in the vicinity of the GSM antennas of a base station. The method replaces the antenna panel with a group of discrete sources of emission. The models represent a very simple tool for the estimation of the electric field. The proposed method for the calculation, requires prior knowledge about the antennas, usually provided by the manufacturer (emitting space in the horizontal plan and vertical plan)

Analysis and comparison between the calculated values and the measured ones concludes that the proposed method provides accurate results for the field close and far from the radio base stations in a given urban area. The error between calculated and measured values is less than $10 \%$.

In an environment with presence of several radio base stations, the intensity of the electrical field, magnetic field and the density of power for different distances from the antenna can be calculated in a short period of time providing confident and accurate results.

Our work in the future will focus in development of an algorithm for the evaluation of the radiation of the field in the presence of several radio base stations by using the proposed theoretical method. This algorithm for the calculation of the electromagnetic field will requires, prior knowledge about the antennas, usually provided by the manufacturer (emitting space in the horizontal plan and vertical plan).

\section{REFERENCES}

[1] Z. Altman, A. Karwowski, M. Wong, J. Wiart and L. Gattoufi, "Dosimetric analysis of base station antennas via simulation and measurements", 15th International Wroclaw Symposium of Electromagnetic Compability EMC'2000, Part-1, pp. 240-244.

[2] A. Karwowski, Z. Altman, "Comparision of simple models for predicting radiofiquency fields in vicinity of base station antennas", Vol. 36, no. 10, pp. 851-861, 11th May 2012.

[3] A. Karwowski and D. Wojcik, "Near field of the GSM base station antennas", Proc. National Telecomunications Symp. KST'2004, Bydgoszcz, vol. 8, pp. 143-152, Sept. 2004.
[4] P. Bemardi, M. Cavagnaro, S. Pisa and E. Piuzzi, "Human exposure to radio base-station antennas in urban environment", IEEE Transactions on Microwave Theory and Techniques, vol. 48, no. 11, pp. 123-132, Nov. 2008.

[5] C. Balanis, “Antenna theory analysis and design”, pp. 212-216, 1998.

[6] A. Marttinez-Gonzalez, A. Fenandez-Pascual, E. Delos, H. Sanchez and D. Hernandez, "Practical procedure for verification of compliance of digital mobile base station to limitations of exposure of the general public to electromagnetic fields", IEEE Proc-MicroWave and Antenna Propagation, vo1.149, no.4, Aug. 2010.

[7] KATHREIN-Werke KG, Technical Information and New Products 790 $2500 \mathrm{MHz}$ Base Station Antennas for Mobile Communications, Catalogue Issue 04/08/10.

[8] M. Barbiroli, C. Carciofi, V. Degli-Esposti and G. Faciasecca, "Evaluation of exposure levels generated by cellular systems: methodology and results", IEEE Transactions on Vehicular Technology, vol. 51, No. 6, pp. 1322-1329, 2009.

[9] Z. Altman, B.Begasse, C. Dale, A. Karwowski, J. Wiart and L. Gattoufi, "Efficient models for base station antennas for human exposure assessment", IEEE Transactions on Electromagnetic Compatibility, vol. 44, No. 4, pp. 588-592, Nov. 2002.

[10] I. Correia, C. Fernandes, G. Carpinteiro and C. Oliveira, "A procedure for estimation and measurement of electromagnetic radiation in the presence of multiple base stations", Instituto Superior Técnico, Lisbon, Portugal, Sept. 2010.

[11] M. Bizzi and P. Gianola, "Electromagnetic fields radiated by GSM antennas", IEEE Transactions on Electromagnetic Compatibility, Electronic Letters, vol. 35, No. 11, pp. 855-857, May 2009.

[12] Available at http://www.narda-sts.de/en/products/emc/

\section{AUTHOR PROFILES}

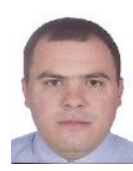

Algenti LALA received his Bachelor of Engineering degree in 1999 and a Masters in Telecommunication Engineering from the Polytechnic University of Tirana (PUT) in 2008. He obtained his Ph.D. from PUT in 2013. From 2008 he has been a Lecturer at the Department of Electronics and Telecommunications, Faculty of Information Technology, at PUT. His research interests lie in the area of electromagnetic field, wave propagation, transmission and antennas. In the latest years he has been focused on methods of calculation of the electromagnetic field in the vicinity of the cellular base stations. He has published several papers in referred Journals and International conference proceedings. He is currently lecturer of several subjects including Measurements of the radio frequencies, Antennas and wave propagation etc. He is scientific tutor of master students, while continuing his scientific research. 